\title{
Timescale of serpentinization reactions on Enceladus
}

\author{
DAMIEN DAVAL ${ }^{1}$, CHOBLET GAËL ${ }^{2}$, CHRISTOPHE \\ SOTIN $^{3}$ AND FRANCOIS J GUYOT ${ }^{4}$
}

\section{${ }^{1}$ LYGHES}

${ }^{2}$ Laboratoire de Géodynamique et Planétologie

${ }^{3}$ Laboratoire de Planétologie et Géodynamique

${ }^{4}$ CNRS-Institut de Minéralogie, de Physique des Matériaux et de Cosmochimie, Sorbonne Université - CNRS - MNHN

Presenting Author: fguyot@mnhn.fr

The Cassini spacecraft demonstrated that Enceladus, a small moon on the outer edge of Saturn's rings, may harbor hydrothermal activity beneath its ice crust $[1,2,3]$. In particular, hydrogen production could result from water-rock interactions in a tidally-heated, water-filled porous rocky core [4]. The lifetime of such reactions is key to assess both the habitability potential of Enceladus and to constrain plausible durations of the active stage in a context where the evolution of the moon is debated [5]. A preliminary study estimated the maximum timescale of serpentinization and reported relatively brief durations (at most a few tens to a couple hundreds of million years) [6]. Here, we investigate plausible rate-limiting mechanisms governing fluidrock interactions that could delay the completion of serpentinization of Enceladus rocky core. In particular, we consider (i) the impact of etch pit nucleation; (ii) the impact of surface layers; (iii) diffusion in nanoporous secondary assemblages; (iv) fluid flow. Our results confirm the former estimate (longest time scale : $300 \mathrm{Myr}$ ) and indicate that fluid flow ultimately sets the tempo for serpentinization. Possible causes to the observed rate of hydrogen emission in the jets of Enceladus will be discussed.

[1] Waite J. H. et al., (2017), Science, 356, 155-159. [2] Hsu, H-W. et al (2015). Nature, 519, 207-210. [3] Sekine, Y. et al. (2015). Nature Comms, 6 (8604). [4] Choblet G. et al., (2017), Nature Astronomy, 1, 841-847. [5] Ida, S. (2019). Science, 364(6445), 1028-1030. [6] Zandanel et al., (2021) in press. 